\title{
Experimental Evaluation on Power Loss of Coreless Double-side Permanent Magnet Synchronous Motor/Generator Applied to Flywheel Energy Storage System
}

\begin{abstract}
Jeong-Man Kim*, Jang-Young Choi ${ }^{\dagger}$ and Sung-Ho Lee**
Abstract - This paper deals with the experimental evaluation on power loss of a double-side permanent magnet synchronous motor/generator (DPMSM/G) applied to a flywheel energy storage system (FESS). Power loss is one of the most important problems in the FESS, which supplies the electrical energy from the mechanical rotation energy, because the power loss decreases the efficiency of energy storage and conversion of capability FESS. In this paper, the power losses of coreless DPMSM/G are separated by the mechanical and rotor eddy current losses in each operating mode. Moreover, the rotor eddy current loss is calculated by the 3-D finite element analysis (FEA) method. The analysis result is validated by separating the power loss as electromagnetic loss and mechanical loss by a spin up/down test.
\end{abstract}

Keywords: Flywheel energy storage system (FESS), Double-side permanent magnet synchronous motor/generator (DPMSM/G), Power loss, Rotor eddy current loss

\section{Introduction}

The flywheel energy storage system (FESS) is an environment-friendly energy storage system which can be used for uninterruptible power supply, power quality improvement, storage of distributed power sources such as solar and wind power, and load leveling. The FESS is an energy storage equipment that supplies electrical energy when needed, after electrical energy is stored into the mechanical rotation energy. In FESS, power losses such as electromagnetic and mechanical losses are mainly a problem because power they decrease the efficiency of energy storage and conversion capability of FESS. Hence, it is essential to evaluate and reduce the power losses occurring in the FESS [1-3].

This paper deals with the experimental evaluation on power loss of a coreless double-side permanent magnet synchronous motor/generator (DPMSM/G) applied to the FESS. In a general PMSM/G, the core losses are generated in the stator core when the PM rotor with a magnetic field is rotated, rotor eddy current losses are generated in the conductive material of the rotor because of the harmonic are magnetic field generated by current, and copper losses generated in the stator windings $[4,5]$. In contrast, the coreless DPMSM/ has only two electromagnetic losses, which are rotor eddy current and copper losses, because the coreless stator winding does not cause the core loss. Using

\footnotetext{
$\dagger \quad$ Corresponding Author: Dept. of Electrical Engineering, Chungnam

National University, Korea. (choi_jy@cnu.ac.kr)

* Dept. of Electrical Engineering, Chungnam National University,

Korea. (jmkim27@cnu.ac.kr)

** Korea Institute of Industrial Technology, Korea. (shlee07@kitech.re.kr)

Received: February 5, 2016; Accepted: August 31, 2016
}

the coreless DPMSM/G the electromagnetic loss that is rotor eddy current loss and copper loss. The mechanical losses such as windage and bearing friction losses are separated and evaluated by a spin up/down test in the idling mode of FESS. In addition, the eddy current losses are predicted by separating power losses in motoring and generating mode. The predicted eddy current losses are compared with 3D FEM analysis result.

\section{Power Loss Definition of Coreless DPMSM/G in FESS}

\subsection{Structural development of the coreless DPMSM/G}

The structure of the coreless DPMSM/G for the FESS is shown in Fig. 1. Fig. 1(a) shows the concept of this model. A double-side PM rotor with a flywheel and a 3phase stator winding are presented in Fig. 1(b) and (c), respectively. The outside and inside rotor possess 16 pieces of Halbach arranged $\mathrm{NdFeB}$ magnet. The coreless stator winding is located between the outside and inside PM rotor. The winding is fixed by a non-magnetic material bobbin.

Table 1. Design Specifications of coreless DPMSM/G

\begin{tabular}{cccc}
\hline \multicolumn{4}{c}{ Main dimensions } \\
\hline Back-EMF constant & $0.124(\mathrm{~V} / \mathrm{rad} / \mathrm{s})$ & Flywheel OD & $170(\mathrm{~mm})$ \\
Moment of inertia & $0.094\left(\mathrm{~kg} \cdot \mathrm{m}^{2}\right)$ & Rotor OD & $53(\mathrm{~mm})$ \\
Rotor Mass & $23.6(\mathrm{~kg})$ & Stack length & $130(\mathrm{~mm})$ \\
\hline \multicolumn{4}{c}{ Material } \\
PM & N35UH & Rotor core & S10C \\
Flywheel & SUS304 & Sleeve & Titanium \\
\hline
\end{tabular}




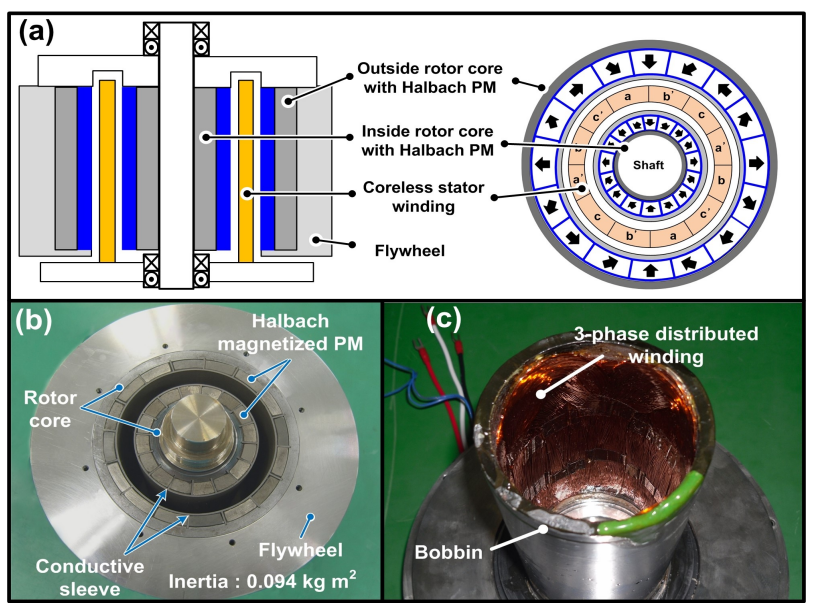

Fig. 1. Structure of coreless DPMSM/G with flywheel: (a) concept of model; (b) double-side PM rotor with flywheel and (c) coreless stator winding

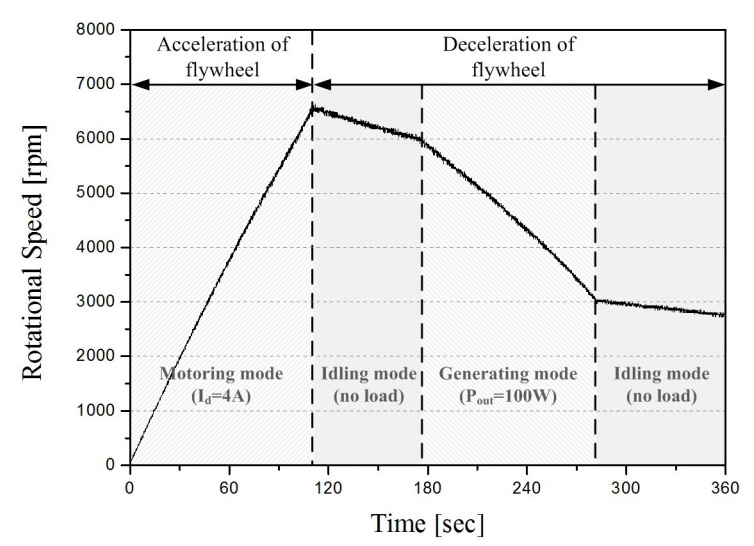

Fig. 2. Dynamic characteristic of the manufactured coreless DPMSM/G according to operating mode

The design specifications of the coreless DPMSM/G are listed in Table 1.

\subsection{Power loss of coreless DPMSM/G in FESS}

Fig. 2 shows the dynamic characteristics of the manufactured coreless DPMSM/G according to the operating modes of the FESS through experimental process. In the motoring mode, electrical energy is converted into mechanical energy. In the idling mode, the flywheel rotor decelerates only because of the mechanical losses. In the generating mode, mechanical energy is converted into electrical energy. The power losses generated in the FESS depending on each operating mode of the coreless $\mathrm{DPMSM} / \mathrm{G}$ can be classified as follows

$$
\begin{aligned}
& P_{\text {loss_mot }}=P_{m}+P_{e}=P_{w d}+P_{f r}+P_{e d d y}+P_{c o} \\
& P_{\text {loss_idl }}=P_{m}=P_{w d}+P_{f r} \\
& P_{\text {loss_gen }}=P_{m}+P_{e}=P_{w d}+P_{f r}+P_{\text {eddy }}+P_{c o}
\end{aligned}
$$
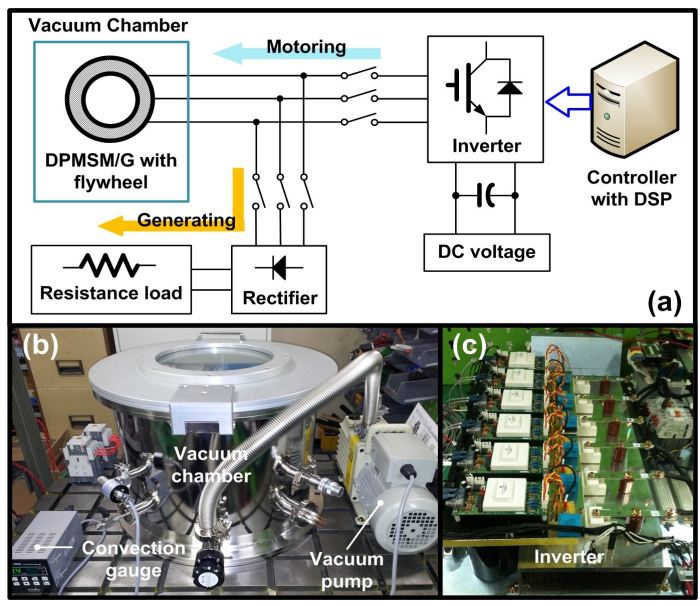

Fig. 3. Experiment set (a) Configuration of FESS system, (b) Vacuum chamber and (c) PWM inverter

where $P_{\text {loss_mot }}, P_{\text {loss_idl, }}$, and $P_{\text {loss_gen }}$ are the power losses in the motoring, idling and generating modes, respectively. $P_{m}$ and $P_{e}$ are mechanical and electromagnetic losses, respectively. $P_{w d}$ and $P_{f}$ are the winding and bearing friction losses, respectively. $P_{e d d y}$ and $P_{c o}$ are the rotor eddy current and copper losses, respectively.

\section{Experimental Approach on Power Loss of Coreless DPMSM/G in FESS}

\subsection{Experiment set}

The experiment set of the coreless DPMSM/G for the FESS is shown in Fig. 3. Fig. 3(a) shows the configuration of the FESS. A vacuum chamber and a PWM inverter are presented in Fig. 3(b) and (c), respectively.

\subsection{Mechanical losses in the idling mode}

Fig. 4(a) shows the speed characteristics of the coreless DPMSM/G by the spin down test in the idling mode. The mechanical losses can be obtained from this deceleration curve because the deceleration in the idling mode is caused only by the mechanical losses. The windage loss can be ignored depending on the degree of the vacuum under 7E-4 torr [3].

Thus, mechanical losses can be divided into the windage and the bearing friction losses by operating the FESS with and without a vacuum chamber. The motion equation in the idling mode is expressed as (2). Here, $B_{w d}$ and $B_{f r}$ are windage and bearing friction coefficients, respectively.

$$
J \frac{d \omega_{r m}}{d t}=-\left(B_{w d}+B_{f r}\right) \omega_{r m}=-B \omega_{r m}
$$

As the rotational power is the product of the angular 

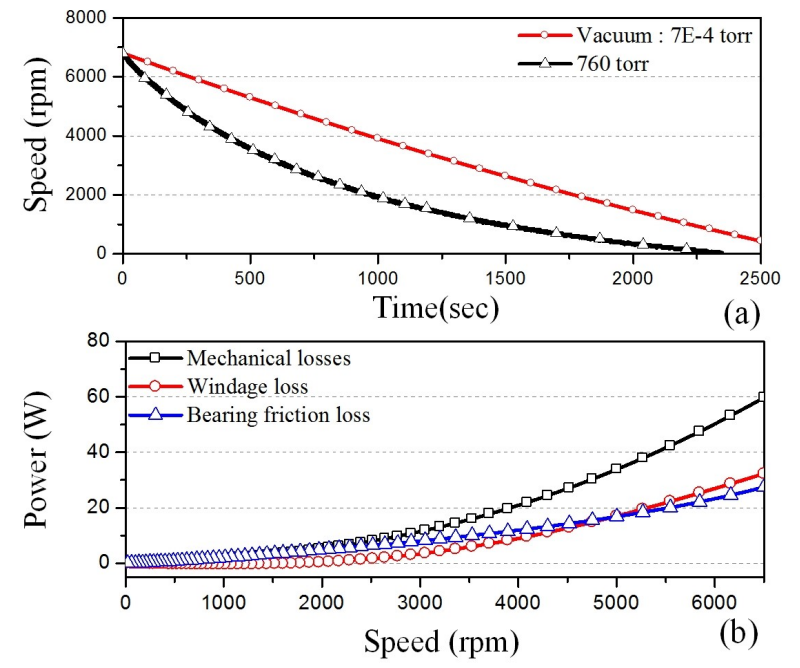

Fig. 4. (a) Result of spin down test in idling mode and (b) mechanical loss of coreless DPMSM/G according to rotational speed

velocity and effective torque for the rotating flywheel, it is defined as (3). In the idling mode, the rotational power represents the mechanical loss as (4).

$$
\begin{gathered}
P_{i d l}=\omega_{r m} J \frac{d \omega_{r m}}{d t} \\
P_{m}=-P_{i d l}=\left(B_{w d}+B_{f r}\right) \omega_{r m}^{2}
\end{gathered}
$$

where $\omega_{r m}$ is the angular velocity, $J$ is the moment of inertia, and $B$ is the coefficient of friction. The mechanical losses of the coreless DPMSM/G according to the rotational speed are classified quantitatively as shown in Fig. 4(b). The mechanical loss obtained in idling mode is also used motoring and generating mode.

\subsection{Power loss in motoring mode}

The motion equation in motoring mode is expressed as (5). $T_{e m}$ and $T_{e d d y}$ are the input electromagnetic torque and the drag torque, respectively, by the rotor eddy current loss. The electromagnetic torque is calculated as (6) [4].

$$
\begin{gathered}
T_{e m}=J \frac{d \omega_{r m}}{d t}+B \omega_{r m}+T_{e d d y} \\
T_{e m}=\frac{3 \omega_{r m} \lambda_{f} i_{q}}{2} \frac{1}{\omega_{r m}}=1.5 \lambda_{f} i_{q}
\end{gathered}
$$

where $\lambda_{f}$ and $i_{q}$ are flux linkage and q-axis current, respectively. In our system, the current control is used to maintain the torque constant. Fig. 5(a) shows the speed characteristics of the coreless DPMSM/G in the motoring mode according to actual q-axis current. Using this acceleration curve, the rotational power is calculated as shown in Fig. 5(b). The rotor eddy current loss $P_{\text {eddy }}$ in
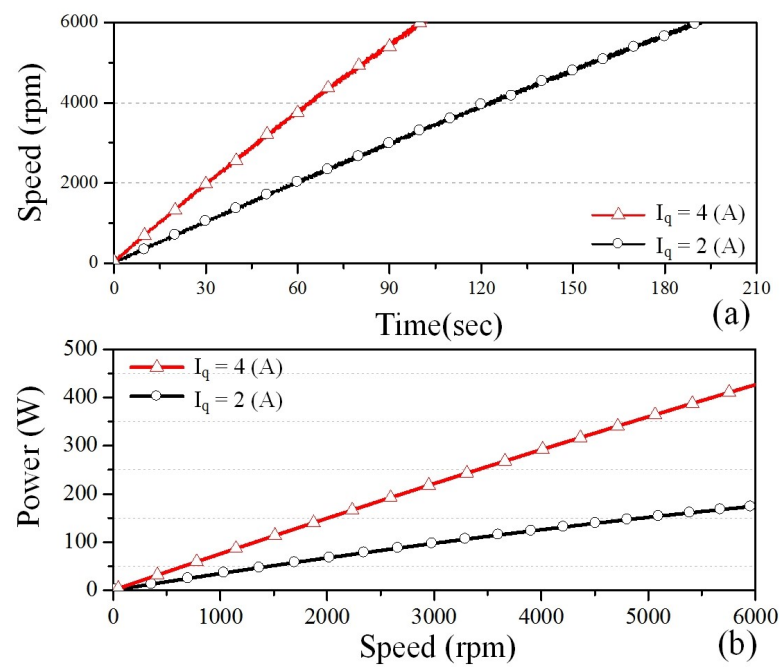

Fig. 5. (a) Result of spin up test in motoring mode and (b) rotational power of coreless DPMSM/G
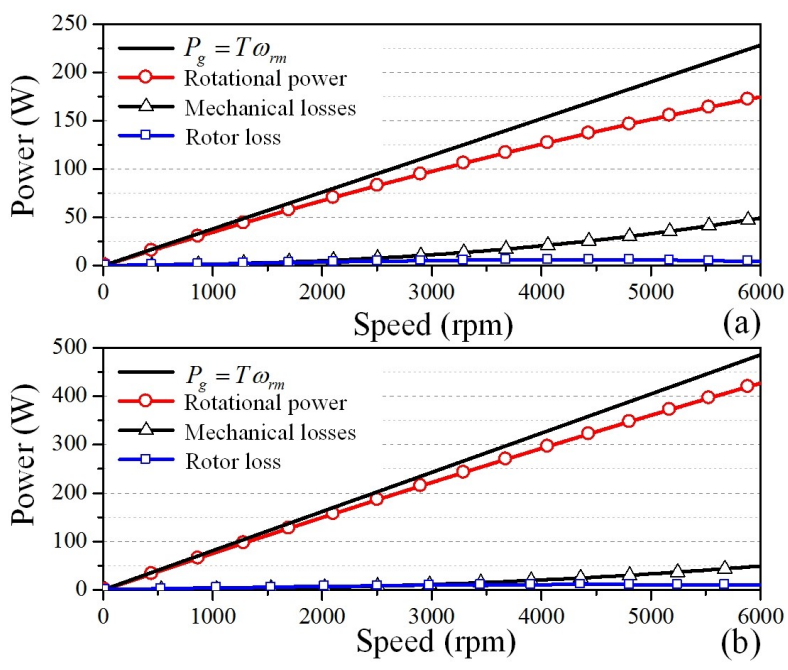

Fig. 6. (a) Results of separated power loss in motoring mode (a) $I_{q}=2[\mathrm{~A}]$ and (b) $I_{q}=4[\mathrm{~A}]$

motoring mode can be obtained from (7).

$$
P_{e d d y}=\omega_{r m} T_{e m}=\omega_{r m}\left(T_{e m}-J \frac{d \omega_{r m}}{d t}-B \omega_{r m}\right)
$$

In contrast, the coreless DPMSM/G has only two electro-magnetic losses, which are rotor eddy current and copper losses, because the coreless stator winding does not cause the core loss. Therefore, the electromagnetic losses can be divided eddy current losses and copper losses in motoring and generating without mechanical losses. This paper was predicted eddy current loss by separating the mechanical losses and copper losses from the measured speed in motoring and generating mode. Fig. 6 shows the characteristics of the power loss divided into the mechanical and rotor eddy current losses according to the 
rotational speed and actual q-axis current.

\subsection{Power losses in the generating mode}

The motion equation in the motoring mode is expressed as (8). $T_{\text {load }}$ is the reverse torque by generating power and copper loss.

$$
T_{\text {load }}=-\left(J \frac{d \omega_{r m}}{d t}+B \omega_{r m}+T_{e d d y}\right)
$$

Fig. 7(a) shows the speed characteristics of the coreless $\mathrm{DPMSM} / \mathrm{G}$ in the generating mode according to the generating power. Using this deceleration curve, the rotational power is calculated as shown in Fig. 7(b). The
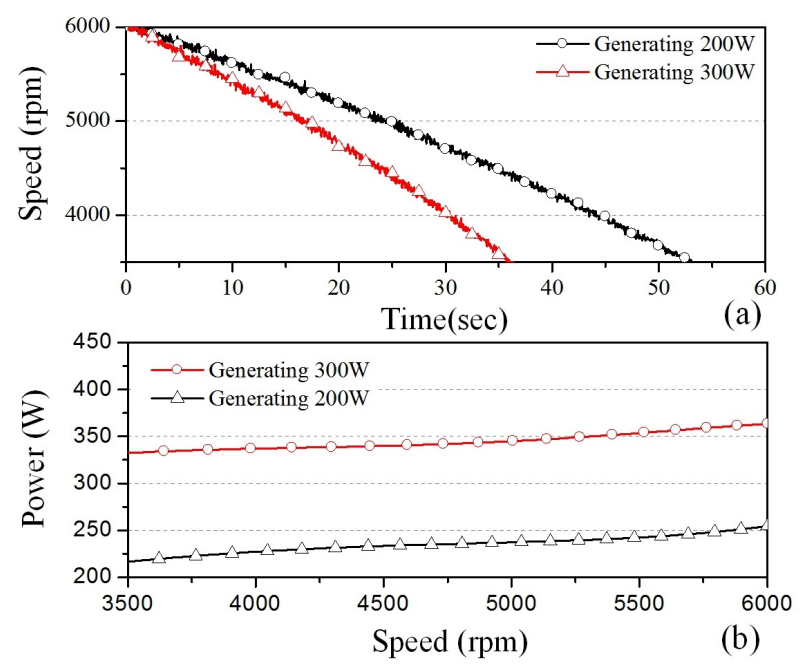

Fig. 7. (a) Result of spin down test in generating mode and (b) rotational power of coreless DPMSM/G
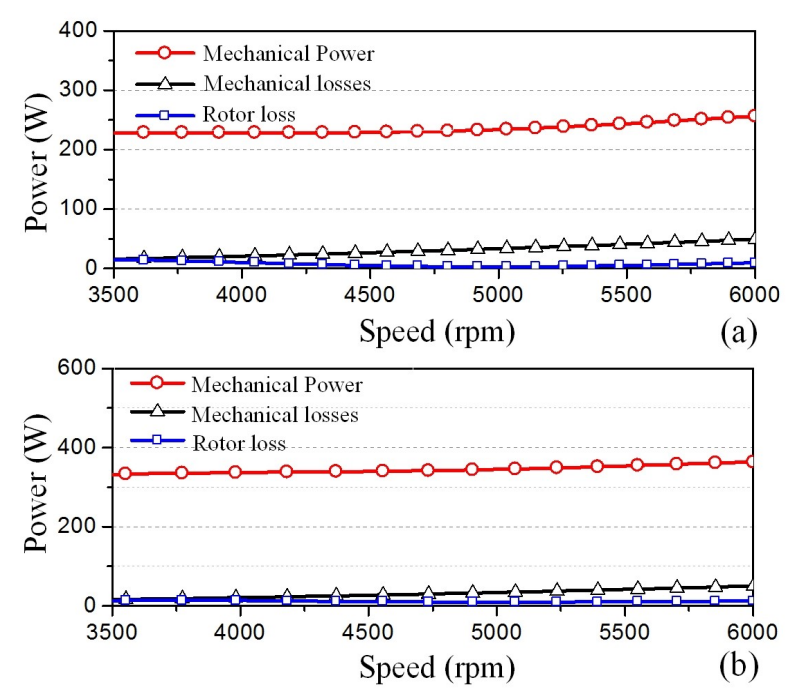

Fig. 8. (a) Result of separated power loss in generating mode according to constant output power: (a) 200 [W] and (b) 300 [W] rotor eddy current loss $P_{\text {edd } y}$ in the generating mode can be obtained as

$$
P_{e d d y}=-\omega_{r m}\left(J \frac{d \omega_{r m}}{d t}+B \omega_{r m}\right)-P_{l o a d}
$$

where $P_{\text {load }}$ is the generating power with copper loss. Fig. 8 shows the characteristics of the power losses divided into mechanical and rotor eddy current losses according to the generating power.

\section{Rotor Eddy Current Loss Analysis and Comparison with Experimental Result}

The rotor eddy current losses in the magnet and conductive sleeve of the coreless DPMSM/G with different operating modes are calculated using the 3-D FEA which is the commercial software Maxwell. In order to minimize the errors in the current waveform, the measured current waveforms are directly used for transient FEA. The measured current waveforms and their FFT analysis results are as shown in Fig. 9 and Fig. 10. The harmonics of the motoring current are caused by the PWM switching frequency [5], and the harmonics of the generating current
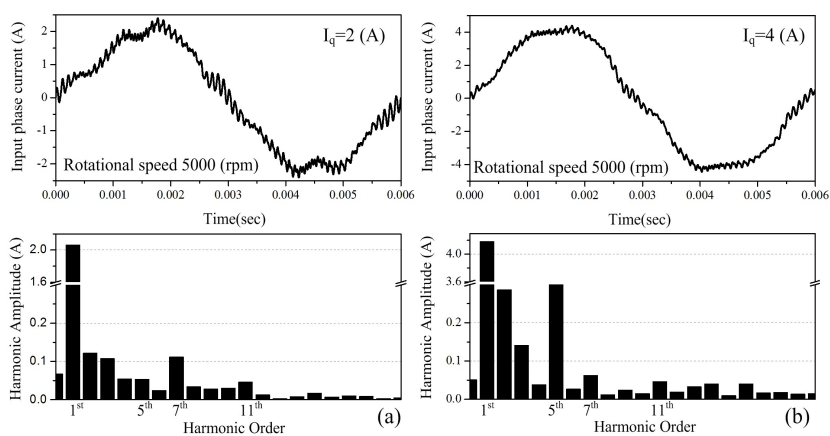

Fig. 9. Motoring current and its FFT analysis result at rotational speed $5000[\mathrm{rpm}]$ (a) $I_{q}=2\left[\right.$ A] and (b) $I_{q}$ $=4[\mathrm{~A}]$
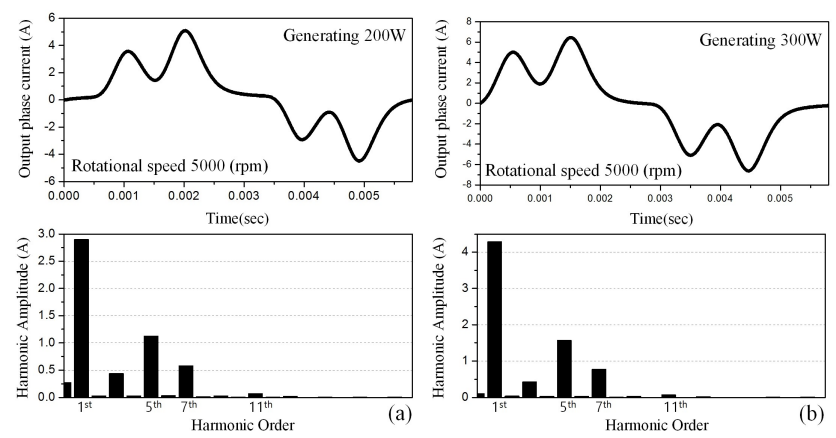

Fig. 10. Generating current and its FFT analysis result at rotational speed 5000 [rpm] (a) 200 [W] and (b) $300[\mathrm{~W}]$ 
are induced due to rectifier load [6]. The rotor eddy current loss is calculated from eddy current density as follows

$$
\begin{aligned}
& I=\int J \cdot d A, \quad J=\left(\sigma+j \omega \varepsilon_{r}\right)(-j \omega A-\nabla \phi) \\
& W_{\text {sleeve+magnet }}=\sum_{n}\left\{\int_{\text {sleeve }+ \text { magnet }} \frac{\left|J_{n}^{2}\right|}{2 \sigma} d v\right\}
\end{aligned}
$$

where $J_{e}$ is the eddy current density in the sleeve or permanent magnet, $V$ is the volume, and $\sigma$ is the electric conductivity.

Fig. 11 shows the eddy current loss distribution obtained by $3 \mathrm{D}$ FEA in motoring mode at rotating speed $5000 \mathrm{rpm}$. It can be seen that the eddy current loss in sleeve is higher than that in magnets. This is because the eddy current in the sleeve acts as a magnetic shield, reducing the amplitude of the varying field to which the magnets are exposed. Table 2 lists the comparison results of the 3D FEA method with the experiment, which is presented in this paper, for

Table 2. Comparison of rotor eddy current loss

\begin{tabular}{c|c|c|c|c|c|c|c|c}
\hline \multirow{2}{*}{$\begin{array}{c}\text { Speed } \\
(\mathrm{rpm})\end{array}$} & \multicolumn{3}{|c|}{ Motoring mode (W) } & \multicolumn{3}{c}{ Generating mode (W) } \\
\cline { 2 - 9 } & Analysis & \multicolumn{2}{c|}{ Experiment } & \multicolumn{2}{c}{ Analysis } & \multicolumn{2}{c}{ Experiment } \\
\cline { 2 - 9 } & $2 \mathrm{~A}$ & $4 \mathrm{~A}$ & $2 \mathrm{~A}$ & $4 \mathrm{~A}$ & 200 & 300 & 200 & 300 \\
\hline 3000 & 2.2 & 3.4 & 3.2 & 5.3 & 3.7 & 6.5 & 4.6 & 8.7 \\
\hline 4000 & 2.7 & 3.8 & 3.8 & 5.8 & 1.2 & 2.3 & 1.6 & 3.4 \\
\hline 5000 & 3.1 & 4.1 & 4.2 & 6.1 & 0.4 & 0.7 & 1.0 & 1.7 \\
\hline
\end{tabular}

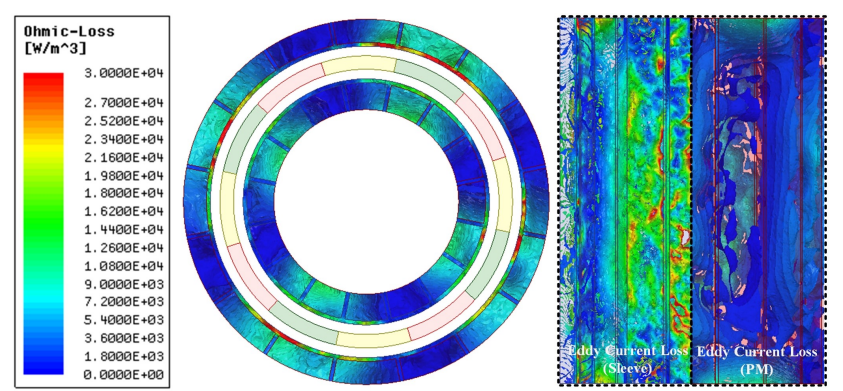

Fig. 11. Rotor eddy current loss distribution obtained by 3D FEA in motoring mode at rotational speed $5000[\mathrm{rpm}]$

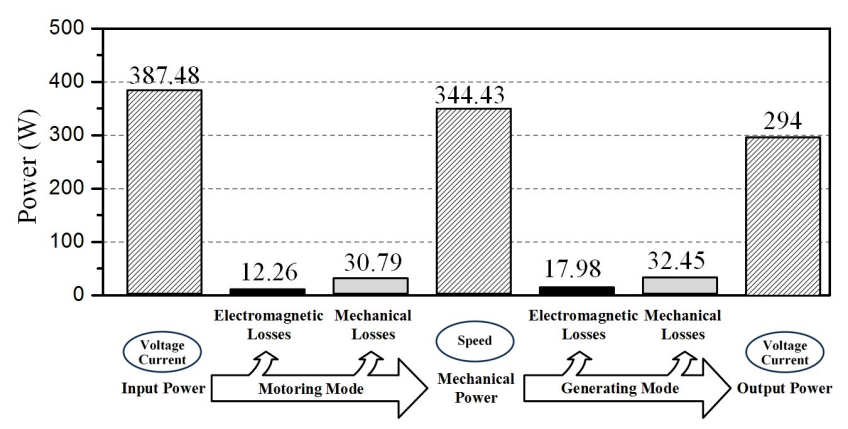

Fig. 12. Power characteristic of FESS at motoring and generating mode the rotor eddy current losses. It can be seen that the predicted eddy current losses are larger than analysis result. This results are a small discrepancy with the values of the eddy current losses between predicted and calculated. Because of the eddy current losses are too low compared to the other losses. However, the prediction of eddy current losses was confirmed the validity of the experimental evaluation method by showing a similar tendency. The coreless DPMSM/G configuration is especially interesting for applications where low idle losses are crucial. Fig. 12 shows the power characteristic of FESS at motoring and generating mode. It is confirmed that the mechanical loss contributes largely to the total energy loss. This can be attributed to the use of ball bearings in our system. In a general application of the FESS, because magnetic bearing is used, the mechanical loss can be extremely reduced.

\section{Conclusion}

This paper presented the experimental evaluation on the power loss of the coreless DPMSM/G applied to the FESS. The power loss was obtained and separated by the spin up / down test according to the operating modes of the FESS. Consequently, the suggested experimental approach can be used to evaluate and validate the power loss of other FESSs. Moreover, because the coreless DPMSM/G is a very efficient electric machine in aspect of the electromagnetic loss, it can be suitably applied to the FESS.

\section{Acknowledgments}

This work was supported by research fund of Chungnam National University.

\section{References}

[1] R. Hebner, J. Beno, and A. Walls, "Flywheel batteries come around again,” IEEE Spectrum, vol. 39, p. 46, 2002.

[2] Jeong-Phil Lee; Nyeon-Ho Jeong; Young-Hee Han; Sang-Chul Han; Se-yong Jung; Byung-Jun Park; TaeHyun Sung, "Assessment of the Energy Loss for SFES With Rotational Core Type PMSM/G", IEEE Trans. Applied Superconductivity, vol.19, Page 20872090, 2009.

[3] J. R. Hull, T. M. Mulcahy, and K. L. Uherka, "Low rotational drag in high-temperature superconducting bearings," IEEE Trans. Applied Superconductivity, vol. 5, no. 2, 1996.

[4] Seok-Myeong Jang, Un-Ho Lee, Dae-Joon You, Jeong-Phil Lee, and Sang-Ho Choi, "Operating Torque Estimation of High-Speed Slotless Brushless DC Machine Considering Power Loss," IEEE Trans. 
Magnetics, vol. 45, pp. 4539-4542, 2009.

[5] Katsumi Yamazaki and Atsushi Abe, "Loss Investigation of Interior Permanent-Magnet Motors Considering Carrier Harmonics and Magnet Eddy Currents," IEEE Trans. Industry Applications, vol. 45, pp. 659-655, 2009.

[6] Yunkai Huang, Jianning Dong, Long Jin, Jianguo Zhu, and Youguang Guo, "Eddy-Current Loss Prediction in the Rotor Magnets of a Permanent Magnet Synchronous Generator With Modular Winding Feeding a Rectifier Load," IEEE Trans. Magnetics, vol. 47, pp. 4203-4206, 2011.

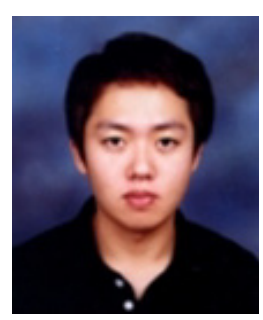

Jeong-Man Kim He received the B.S., and M.S. degrees in electrical engineering from Chungnam National University, Daejeon, Korea, in 2011 and 2013, respectively, $\mathrm{He}$ is currently working for the Ph.D. degree in electrical engineering from Chungnam National University. His current research interests include design and analysis of motor and generator.

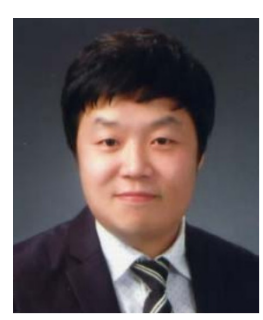

Jang-Young Choi He received his B.S., M.S., and Ph.D. degrees from Chungnam National University in 2003, 2005 , and 2009, respectively. In 2009, he worked as a researcher in Halla Climate Control Corporation. August 2009, Dr. Choi joined as an assistant professor the Department of Electrical Engineering at Chungnam National University, Daejeon, Korea. His interests are in the design, analysis, simulation, and implementation of linear and rotary motors and permanent magnet generators.

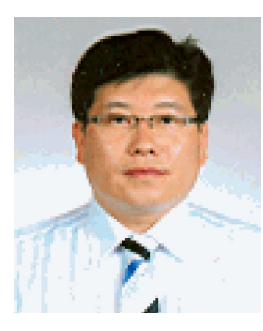

Sung-Ho Lee He received his B.S., M.S., and Ph.D. degrees from Chungnam National University in 1997, 1999, and 2003, respectively. He has worked for LG Electronics as a Senior Engineer. $\mathrm{He}$ is currently with the Automotive Components \& Materials Group, Korea Institute of Industrial Technology. His research interests are ultra high speed machines, wind generators, and Eco machines. 